\title{
THE BOUNDARY BEHAVIOR AND UNIQUENESS OF SOLUTIONS OF THE HEAT EQUATION
}

\author{
BY \\ F. W. GEHRING( ${ }^{(1)}$
}

1. Introduction. In this paper we consider some questions on the boundary behavior and uniqueness of solutions of the one-dimensional heat equation in the infinite strip $0<t<c$ and in the half plane $0<t<\infty$. For convenience we adopt the following notation.

Suppose that $u=u(x, t)$ is defined over some domain $\delta$ in the $x t$-plane. We say that $u \in H$ in $\delta$ if $u$ has continuous second partial derivatives and if $u_{t}=u_{x x}$ at each point of $\delta$. We say that $u \in H^{+}$in $\delta$ if, in addition, $u \geqq 0$ in $\delta$ and that $u \in H^{\Delta}$ in $\delta$ if $u=u_{1}-u_{2}$ where $u_{1}, u_{2} \in H^{+}$in $\delta$. Clearly $H^{+} \subset H^{\Delta}$.

By a well known theorem due to Widder [19] $u \in H^{\Delta}$ in $0<t<c$ if and only if $u$ has the representation

$$
u(x, t)=\int_{-\infty}^{\infty} k(x-y, t) d \alpha(y)
$$

in $0<t<c$, where $\alpha=\alpha(x)$ has bounded variation over each finite interval, where the integral is absolutely convergent in this strip, and where $k(x, t)$ is the Poisson kernel

$$
k(x, t)=\frac{1}{(4 \pi t)^{1 / 2}} e^{-x^{2} / 4 t} .
$$

(See also [7].) If we assume, as we clearly may, that $\alpha$ is normalized, i.e.

$$
\alpha(x)=\frac{\alpha(x+0)+\alpha(x-0)}{2}
$$

for all $x$, then

$$
\alpha\left(x_{2}\right)-\alpha\left(x_{1}\right)=\lim _{t \rightarrow 0+} \int_{x_{1}}^{x_{2}} u(y, t) d y
$$

for all $x_{1}, x_{2}$. (See [3] or [21]. This is also a simple consequence of Theorem 1.) Hence with each $u \in H^{\Delta}$ in $0<t<c$ we can associate an $\alpha$, unique except for an additive constant, satisfying (1.1), (1.2), and (1.3). If we think of $u(x, t)$ as the temperature of an infinite insulated rod, extended along the $x$-axis, at the point $x$ of the rod and at time $t$, then $\alpha(x)$ represents the heat distribution in the rod at time $t=0$.

Received by the editors February 18, 1959.

(1) This work was in part supported by a Fellowship from the Guggenheim Foundation. 
Solutions of the heat equation and harmonic functions have many similar properties and in this paper we obtain the analogues, for functions in $H^{\Delta}$ over $0<t<c$, of several well known theorems concerning functions which are harmonic in a half plane or, alternatively, which are harmonic in the unit circle. In $\$ 2$ we consider various forms of the Fatou theorem and obtain information on the behavior of $u(x, t)$ as $(x, t)$ approaches $\left(x_{0}, 0\right)$ from information on the behavior of $\alpha(x)$ near $x_{0}$. A simple example shows that, in general, it is not possible to invert the results of $\$ 2$ and obtain information on the behavior of $\alpha(x)$ near $x_{0}$ from the behavior of $u(x, t)$ near $\left(x_{0}, 0\right)$. However, in $\S 3$ and $\S 4$, we restrict our attention to the important subclass of functions in $\mathrm{H}^{+}$and we obtain the "corrected converses" or Tauberian theorems corresponding to the Abelian theorems of $\$ 2$. (For functions in $H^{\Delta}$ and in $H^{+}$ over $0<t<\infty$ we establish analogous relations between the behavior of $u(x, t)$ as $t \rightarrow \infty$ and the behavior of $\alpha(x)$ for large $x$.) In $\$ 5$ we list some uniqueness theorems and in $\$ 6$ we give an analogue for an inequality due to Fejér and F. Riesz.

2. Fatou theorem. For each $a>0$ we let $S\left(a, x_{0}\right)$ and $P\left(a, x_{0}\right)$ denote, respectively, the following sector and parabolic domains:

$$
\begin{aligned}
& S\left(a, x_{0}\right)=\left\{(x, t):\left|x-x_{0}\right|<a t, t>0\right\}, \\
& P\left(a, x_{0}\right)=\left\{(x, t):\left|x-x_{0}\right|<a t^{1 / 2}, t>0\right\} .
\end{aligned}
$$

From the identity

$$
\int_{-\infty}^{\infty}\left|\frac{x}{2 t^{1 / 2}}\right|^{n} k(x, t) d x=\frac{1}{\pi^{1 / 2}} \Gamma\left(\frac{n+1}{2}\right), \quad n>-1,
$$

it is not difficult to show that, for each $a>0$, there exists a constant $C_{1}=C_{1}(a)$ $<\infty$ such that

$$
\int_{-\infty}^{\infty}\left|y k_{y}(x-y, t)\right| d y \leqq C_{1}, \quad \int_{-\infty}^{\infty}\left|y k_{y y}(x-y, t)\right| d y \leqq \frac{C_{1}}{t^{1 / 2}}
$$

for all $(x, t) \in P(a, 0)$.

For functions in $H^{\Delta}$ we have the following "localization" theorem.

Lemma 1. Suppose that $u \in H^{\Delta}$ in $0<t<c$ and that $d>0$. Then, for each $x_{0}$,

$$
\int_{\left|y-x_{0}\right|>d} k(x-y, t) d \alpha(y)=o(1), \int_{\left|y-x_{0}\right|>d} k_{x}(x-y, t) d \alpha(y)=o\left(\frac{1}{t^{1 / 2}}\right)
$$

as $(x, t) \rightarrow\left(x_{0}, 0\right), t>0$, and

$$
\begin{aligned}
& \int_{\left|y-x_{0}\right|<d} k(x-y, t) d \alpha(y)=O\left(\frac{1}{t^{1 / 2}}\right), \\
& \int_{\left|y-x_{0}\right|<d} k_{x}(x-y, t) d \alpha(y)=O\left(\frac{1}{t}\right)
\end{aligned}
$$


as $t \rightarrow \infty$, uniformly in $x$.

Proof. For the first part of (2.2) fix $0<b<c$. The inequalities $\left|x-x_{0}\right|$ $<d / 2,\left|y-x_{0}\right|>d, 0<t<b / 8$ imply that

$$
k(x-y, t) \leqq \frac{1}{(4 \pi t)^{1 / 2}} e^{-d^{2} / 32 t} e^{-\left(x_{0}-y\right)^{2} / 4 b}=C_{2} k(d, 8 t) k\left(x_{0}-y, b\right)
$$

where $C_{2}=(32 \pi b)^{1 / 2}$. Hence we obtain

$$
\left|\int_{\left|y-x_{0}\right|>d} k(x-y, t) d \alpha(y)\right| \leqq C_{2} k(d, 8 t) \int_{-\infty}^{\infty} k\left(x_{0}-y, b\right)|d \alpha(y)|
$$

for $\left|x-x_{0}\right|<d / 2,0<t<b / 8$, and, since $k(d, 8 t) \rightarrow 0$ as $t \rightarrow 0$, the desired conclusion follows directly. A similar argument yields the second part of (2.2), and (2.3) is an immediate consequence of the inequalities,

$$
k(x, t) \leqq \frac{1}{(4 \pi t)^{1 / 2}}, \quad\left|k_{x}(x, t)\right| \leqq \frac{1}{(4 \pi)^{1 / 2} t}
$$

for all $(x, t)$ in $0<t<\infty$.

Functions in $H^{\Delta}$ satisfy the following order condition near the line $t=0$.

Lemma 2. Suppose that $u \in H^{\Delta}$ in $0<t<c$. If $\alpha$ is continuous at $x_{0}$, then

$$
\cdot u(x, t)=o\left(\frac{1}{t^{1 / 2}}\right), \quad u_{x}(x, t)=o\left(\frac{-1}{t}\right)
$$

as $(x, t) \rightarrow\left(x_{0}, 0\right), t>0$. If $\alpha$ has a jump of $\lambda$ at $x_{0}$, then, for each $a>0$,

$$
u(x, t) \sim \frac{\lambda}{(4 \pi t)^{1 / 2}}, \quad u_{x}(x, t)=o\left(\frac{1}{t}\right)
$$

as $(x, t) \rightarrow\left(x_{0}, 0\right),(x, t) \in S\left(a, x_{0}\right)$.

Proof. If $\alpha$ is continuous at $x_{0}$, we can find a $d>0$ such that

$$
\int_{\left|\nu-x_{0}\right| \leq d}|d \alpha(y)|<\epsilon .
$$

Next we can differentiate both sides of (1.1) [19] and, with (2.2) and (2.4), we conclude that

$$
|u(x, t)| \leqq \frac{\epsilon}{(4 \pi t)^{1 / 2}}+o(1), \quad\left|u_{x}(x, t)\right| \leqq \frac{\epsilon}{(4 \pi)^{1 / 2} t}+o\left(\frac{1}{t^{1 / 2}}\right)
$$

as $(x, t) \rightarrow\left(x_{0}, 0\right), t>0$. Hence (2.5) follows. If $\alpha$ has a jump of $\lambda$ at $x_{0}$, we write $\alpha=\alpha_{c}+\alpha_{j}$, where $\alpha_{c}$ is continuous at $x_{0}$ and where $\alpha_{j}$ is constant except for a jump of $\lambda$ at $x_{0}$. Then 


$$
u(x, t)=\int_{-\infty}^{\infty} k(x-y, t) d \alpha_{c}(y)+\lambda k\left(x-x_{0}, t\right),
$$

and (2.6) follows from (2.5).

We consider next the following two versions of the Fatou theorem for functions in $H^{\Delta}$. The $(x, t) \rightarrow\left(x_{0}, 0\right)$ parts of each of these results are known [15] but proofs are included for the sake of completeness. We let $D \alpha\left(x_{0}\right)$ denote the symmetric derivative of $\alpha$ at $x_{0}$, i.e.

$$
D \alpha\left(x_{0}\right)=\lim _{h \rightarrow 0} \frac{\alpha\left(x_{0}+h\right)-\alpha\left(x_{0}-h\right)}{2 h} .
$$

Theorem 1. Suppose that $u \in H^{\Delta}$ in $0<t<c$. If $D \alpha\left(x_{0}\right)=A$, then, for each $a>0$,

$$
u(x, t) \rightarrow A
$$

as $(x, t) \rightarrow\left(x_{0}, 0\right),(x, t) \in S\left(a, x_{0}\right)$. Suppose that $u \in H^{\Delta}$ in $0<t<\infty$. If

$$
\frac{\alpha\left(x_{0}+x\right)-\alpha\left(x_{0}-x\right)}{2 x} \rightarrow A
$$

as $x \rightarrow \infty$, then $u\left(x_{0}, t\right) \rightarrow A$ as $t \rightarrow \infty$.

Theorem 2. Suppose that $u \in H^{\Delta}$ in $0<t<c$. If $\alpha^{\prime}\left(x_{0}\right)=A$, then, for each $a>0$,

$$
u(x, t) \rightarrow A, \quad u_{x}(x, t)=o\left(\frac{1}{t^{1 / 2}}\right)
$$

as $(x, t) \rightarrow\left(x_{0}, 0\right),(x, t) \in P\left(a, x_{0}\right)$. Suppose that $u \in H^{\Delta}$ in $0<t<\infty$. If

$$
\frac{\alpha\left(x_{0}+x\right)-\alpha\left(x_{0}\right)}{x} \rightarrow A
$$

as $|x| \rightarrow \infty$, then, for each $a>0,(2.8)$ holds as $t \rightarrow \infty,(x, t) \in P\left(a, x_{0}\right)$.

Proof of Theorem 1. If we replace $\alpha(x)$ by $\alpha(x)-\alpha\left(x_{0}\right)$ and $x$ by $x-x_{0}$, we see we can assume that $\alpha\left(x_{0}\right)=0$ and that $x_{0}=0$. The hypotheses for the first part of the theorem are then that

$$
\frac{\alpha(x)-\alpha(-x)}{2 x} \rightarrow A
$$

as $x \rightarrow 0+$. If we let

$$
z=y^{2}, \quad s=\frac{1}{4 t}, \quad \beta(z)=\alpha(y)-\alpha(-y),
$$

we can write 


$$
u(0, t)=\int_{-\infty}^{\infty} k(y, t) d \alpha(y)=\left(\frac{s}{\pi}\right)^{1 / 2} \int_{0}^{\infty} e^{-s z} d \beta(z) .
$$

Because of (2.2), we can assume that $\alpha(x)$ is constant for large $x$ and hence that the Laplace integral in (2.11) converges for $0<s<\infty$. From (2.9) we see that

$$
\frac{\beta(z)}{z^{1 / 2}} \rightarrow 2 A
$$

as $z \rightarrow 0+$, and applying a well known Abelian theorem for the Laplace transform $[20$, p. 182] we conclude that

$$
\left(\frac{s}{\pi}\right)^{1 / 2} \int_{0}^{\infty} e^{-s z} d \beta(z) \rightarrow A
$$

as $s \rightarrow \infty$. Hence

$$
u(0, t) \rightarrow A
$$

as $t \rightarrow 0+$, and the convergence in $S(a, 0)$ is an immediate consequence of Lemma 2.

The hypotheses for the second part of Theorem 1 imply that the Laplace integral in (2.11) converges for $0<s<\infty$ and that (2.12) holds as $z \rightarrow \infty$. Appealing to the same Abelian theorem we obtain (2.13) as $s \rightarrow 0+$ and this completes the proof.

Proof of Theorem 2. As in the proof for Theorem 1 we can assume that $\alpha\left(x_{0}\right)=0, x_{0}=0$. Furthermore, since

$$
u(x, t)=\int_{-\infty}^{\infty} k(x-y, t) d\{\alpha(y)-A y\}+A,
$$

we need only consider the case where $A=0$.

The hypotheses for the first part of Theorem 2 imply we can find a $d>0$ such that

$$
|\alpha(x)| \leqq \epsilon|x|,
$$

for $|x| \leqq d$. Then (2.2) and an integration by parts yield

$$
\begin{aligned}
& u(x, t)=-\int_{|y| \leqq d} k_{y}(x-y, t) \alpha(y) d y+o(1), \\
& u_{x}(x, t)=+\int_{|y| \leqq d} k_{y y}(x-y, t) \alpha(y) d y+o\left(\frac{1}{t^{1 / 2}}\right)
\end{aligned}
$$

as $(x, t) \rightarrow(0,0), t>0$. Appealing to (2.14) and (2.1), we conclude that 


$$
\begin{aligned}
& |u(x, t)| \leqq \epsilon \int_{-\infty}^{\infty}\left|y k_{y}(x-y, t)\right| d y+o(1) \leqq \epsilon C_{1}+o(1), \\
& \left|u_{x}(x, t)\right| \leqq \epsilon \int_{-\infty}^{\infty}\left|y k_{y y}(x-y, t)\right| d y+o\left(\frac{1}{t^{1 / 2}}\right) \leqq \frac{\epsilon C_{1}}{t^{1 / 2}}+o\left(\frac{1}{t^{1 / 2}}\right)
\end{aligned}
$$

as $(x, t) \rightarrow(0,0),(x, t) \in P(a, 0)$. This completes the argument.

The hypotheses for the second part of Theorem 2 imply that (2.14) holds for $|x| \geqq d$. From (2.3) and integration by parts, we obtain (2.15) as $t \rightarrow \infty$, uniformly in $x$, with the integration taken over the range $|y| \geqq d$. The proof is then completed as before.

We will require the following results in $\$ \S 3-4$ and in $\$ 5$, respectively. They can be obtained with trivial modifications of the argument used in the proof of Theorem 2 .

Corollary 1. Suppose that $u \in H^{\Delta}$ in $0<t<c$. If

$$
\frac{\alpha\left(x_{0}+x\right)-\alpha\left(x_{0}\right)}{x}=O(1)
$$

as $x \rightarrow 0$, then, for each $a>0$,

$$
u(x, t)=O(1), \quad u_{x}(x, t)=O\left(\frac{1}{t^{1 / 2}}\right)
$$

as $(x, t) \rightarrow\left(x_{0}, 0\right),(x, t) \in P\left(a, x_{0}\right)$. Suppose that $u \in H^{\Delta}$ in $0<t<\infty$. If (2.16) holds as $|x| \rightarrow \infty$, then, for each $a>0,(2.17)$ holds as $t \rightarrow \infty,(x, t) \in P\left(a, x_{0}\right)$.

Corollary 2. Suppose that $u \in H^{\Delta}$ in $0<t<c$. If $\alpha^{\prime}\left(x_{0}\right)=\infty(-\infty)$, then

$$
u\left(x_{0}, t\right) \rightarrow \infty(-\infty)
$$

as $t \rightarrow 0+$.

3. Converses for the Fatou theorem. We begin with an example which shows that, in general, Theorems 1 and 2 cannot be inverted (cf. [12, p. 246]).

Theorem 3. There exists a function $u \in H^{\Delta}$ in $0<t<\infty$ such that, for each $a>0$,

$$
u(x, t) \rightarrow 0, \quad u_{x}(x, t)=o\left(\frac{1}{t^{1 / 2}}\right)
$$

as $(x, t) \rightarrow(0,0),(x, t) \in P(a, 0)$, and as $t \rightarrow \infty,(x, t) \in P(a, 0)$, and such that

$$
\lim \inf \frac{\alpha(x)-\alpha(-x)}{2 x}<\lim \sup \frac{\alpha(x)-\alpha(-x)}{2 x}
$$

as $x \rightarrow 0+$ and as $x \rightarrow \infty$. 
Proof. Let $\alpha$ be the normalized function defined as follows:

$$
\alpha(x)= \begin{cases}2^{n} & 2^{n}<x<2^{n}+b_{n}, \\ 2^{n-1} & x=2^{n}, 2^{n}+b_{n}, \quad n=0, \pm 1, \pm 2, \cdots \\ 0 & \text { everywhere else. }\end{cases}
$$

The $b_{n}$ are chosen so that $0<b_{n}<2^{n}$ and so that

$$
\sum_{n=-\infty}^{\infty} 2^{-n} b_{n}<\infty
$$

Then (3.2) holds as $x \rightarrow 0+$ and as $x \rightarrow \infty$. With the Law of the Mean we obtain

$$
u(x, t)=\int_{-\infty}^{\infty} k(x-y, t) d \alpha(y)=\sum_{n=-\infty}^{\infty} 2^{n} b_{n} k_{x}\left(x-y_{n}, t\right),
$$

where $2^{n}<y_{n}<2^{n}+b_{n}<2^{n+1}$. Fix $a>0$. It is not difficult to see that

$$
\sup _{(x, t) \in P(a, 0)}\left|k_{x}(x-2 y, t)\right|=\frac{1}{4} \sup _{(x, t) \in P(a, 0)}\left|k_{x}(x-y, t)\right|<\infty
$$

for each $y \neq 0$. Hence for $(x, t) \in P(a, 0)$ we have

$$
\begin{aligned}
\left|k_{x}\left(x-y_{n}, t\right)\right| & \leqq \sup _{(x, t) \in P(a, 0)}\left|k_{x}\left(x-y_{n}, t\right)\right|=4^{-n} \sup _{(x, t) \in P(a, 0)}\left|k_{x}\left(x-2^{-n} y_{n}, t\right)\right| \\
& \leqq 4^{-n} \sup _{(x, t) \in P(a, 0) ; 1<\nu<2}\left|k_{x}(x-y, t)\right|=4^{-n} C_{3}
\end{aligned}
$$

for $n=0, \pm 1, \pm 2, \cdots$, where $C_{3}=C_{3}(a)<\infty$. From (3.3) and (3.5) we conclude that the series in (3.4) is uniformly convergent in $P(a, 0)$. Since

$$
k_{x}\left(x-y_{n}, t\right) \rightarrow 0
$$

as $(x, t) \rightarrow(0,0), t>0$, and as $t \rightarrow \infty$, uniformly in $x$, the first part of (3.1) follows directly. The above argument can be modified to show that the series

$$
t^{1 / 2} u_{x}(x, t)=\sum_{n=-\infty}^{\infty} 2^{n} b_{n} t^{1 / 2} k_{x x}\left(x-z_{n}, t\right), \quad 2^{n}<z_{n}<2^{n}+b_{n}
$$

is uniformly convergent in $P(a, 0)$, and the second part of (3.1) follows from the fact that

$$
t^{1 / 2} k_{x x}\left(x-z_{n}, t\right) \rightarrow 0
$$

as $(x, t) \rightarrow(0,0), t>0$, and as $t \rightarrow \infty$, uniformly in $x$.

Theorem 3 shows that the unrestricted converses for Theorems 1 and 2 are false. However, if we consider the important subclass $\mathrm{H}^{+}$, the situation is quite different and we have the following results. (Cf. [6] and [12].) 
Theorem 4. Suppose that $u \in H^{+}$in $0<t<c$. If, for some $a$,

$$
u\left(x_{0}+a t, t\right) \rightarrow A
$$

as $t \rightarrow 0+$, then $D \alpha\left(x_{0}\right)=A$. Suppose that $u \in H^{+}$in $0<t<\infty$. If

$$
u\left(x_{0}, t\right) \rightarrow A
$$

as $t \rightarrow \infty$, then

$$
\frac{\alpha\left(x_{0}+x\right)-\alpha\left(x_{0}-x\right)}{2 x} \rightarrow A
$$

as $x \rightarrow \infty$.

Theorem 5. Suppose that $u \in H^{+}$in $0<t<c$. If, for some $a$ and $b$,

$$
u\left(x_{0}+a t^{1 / 2}, t\right) \rightarrow A, \quad u\left(x_{0}+b t^{1 / 2}, t\right) \rightarrow A,
$$

$a \neq b$,

as $t \rightarrow 0+$, then $\alpha^{\prime}\left(x_{0}\right)=A$. Suppose that $u \in H^{+}$in $0<t<\infty$. If, for some $a$ and $b$, (3.7) holds as $t \rightarrow \infty$, then

$$
\frac{\alpha\left(x_{0}+x\right)-\alpha\left(x_{0}\right)}{x} \rightarrow A
$$

as $|x| \rightarrow \infty$.

Theorem 6. Suppose that $u \in H^{+}$in $0<t<c$. If, for some $a$ and $b$,

$$
u\left(x_{0}+a t^{1 / 2}, t\right) \rightarrow A, \quad u_{x}\left(x_{0}+b t^{1 / 2}, t\right)=o\left(\frac{1}{t^{1 / 2}}\right)
$$

as $t \rightarrow 0+$, then $\alpha^{\prime}\left(x_{0}\right)=A$. Suppose that $u \in H^{+}$in $0<t<\infty$. If, for some a and $b$, (3.9) holds as $t \rightarrow \infty$, then (3.8) holds as $|x| \rightarrow \infty$.

Proof of Theorem 4. Assume that $\alpha\left(x_{0}\right)=0, x_{0}=0$, and consider the first part of Theorem 4. Fronı (3.6) and Lemma 2 we see that

$$
u(0, t) \rightarrow A
$$

as $t \rightarrow 0+$. Now define $z, s, \beta$, as in (2.10). The hypothesis that $u \in H^{+}$implies that $\beta$ is nondecreasing and, with (2.2), we can assume that the Laplace integral in (2.11) is convergent for $0<s<\infty$. From the above we conclude that (2.13) holds as $s \rightarrow \infty$ and we obtain (2.12), as $z \rightarrow 0+$, from a well known Tauberian theorem for the Laplace transform [20, p. 192]. This completes the proof for the first part of Theorem 4 . The argument for the second part is very similar.

The proofs for Theorems 5 and 6 are more complicated and depend upon a number of preliminary results.

We begin with some definitions [8]. We say that $f \in M$ if $f$ is continuous in $0<x<\infty$ and if 


$$
\sum_{n=-\infty}^{\infty} \max _{2^{n} \leqq x \leqq 2^{n+1}}|x f(x)|<\infty .
$$

For such functions we adopt the notation

$$
F(\mu)=\int_{0}^{\infty} f(x) x^{i \mu} d x, \quad F=F(0),
$$

where $\mu$ is real. We say that $\alpha \in V$ if $\alpha$ has bounded variation over each finite interval in $0<x<\infty$ and if

$$
\int_{x}^{2 x} \frac{|d \alpha(y)|}{y}
$$

is bounded for $\left({ }^{2}\right) 0<x<\infty$.

We have next the following variant of the Wiener Tauberian theorem [6, Corollary 1].

Lemma 3. Suppose that $f_{1}, f_{2}, g_{1}, g_{2} \in M$, that $\alpha, \beta \in V$, that $\alpha, \beta$ are monotone, that $\alpha(0)=\beta(0)=0$, and that

$$
F_{1}(\mu) G_{2}(\mu)-F_{2}(\mu) G_{1}(\mu) \neq 0
$$

for all real $\mu$. If

$$
\begin{aligned}
& \frac{1}{s} \int_{0}^{\infty} f_{1}(y / s) d \alpha(y)+\frac{1}{s} \int_{0}^{\infty} f_{2}(y / s) d \beta(y) \rightarrow A, \\
& \frac{1}{s} \int_{0}^{\infty} g_{1}(y / s) d \alpha(y)+\frac{1}{s} \int_{0}^{\infty} g_{2}(y / s) d \beta(y) \rightarrow B
\end{aligned}
$$

as $s \rightarrow 0+(\infty)$, then

$$
\frac{\alpha(x)}{x} \rightarrow \frac{A G_{2}-B F_{2}}{F_{1} G_{2}-F_{2} G_{1}}, \quad \frac{\beta(x)}{x} \rightarrow \frac{B F_{1}-A G_{1}}{F_{1} G_{2}-F_{2} G_{1}}
$$

as $x \rightarrow 0+(\infty)$.

In the proof of Theorems 5 and 6 we use the following result to show that condition (3.11) is satisfied for a special set of functions.

Lemma 4. For real $x$ and $\mu$ let

$$
\begin{aligned}
& \theta_{1}(x)=\theta_{1}(x, \mu)=\int_{0}^{\infty} e^{-(y-x)^{2}} y^{i \mu} d y, \\
& \theta_{2}(x)=\theta_{2}(x, \mu)=\int_{0}^{\infty} e^{-(y+x)^{2}} y^{i \mu} d y .
\end{aligned}
$$

(2) If $f \in M$ and $\alpha \in V$, then the integral $\int_{0}^{\infty} f(x) d \alpha(x)$ is absolutely convergent. In particular if $f \in M$, then $f$ is Lebesgue integrable over $0<x<\infty$. 
Then, for each fixed $\mu$,

$$
\theta_{1}(a) \theta_{2}(b)-\theta_{2}(a) \theta_{1}(b) \neq 0
$$

for all $a$ and $b, a \neq b$, and

$$
\theta_{1}(a) \theta_{2}^{\prime}(b)-\theta_{2}(a) \theta_{1}^{\prime}(b) \neq 0
$$

or all $a$ and $b$.

Proof. If $w=w(x)$ is any solution of the differential equation

$$
w^{\prime \prime}+2 x w^{\prime}-2 i \mu w=0,
$$

then a standard calculation yields

$$
\left.e^{x^{2} \bar{w} w w^{\prime}}\right]_{a}^{b}=\int_{a}^{b} e^{x^{2}}\left|w^{\prime}\right|^{2} d x+2 i \mu \int_{a}^{b} e^{x^{2}}|w|^{2} d x
$$

for real $a$ and $b$, where the bar denotes complex conjugate. (Cf. [10, p. 509].) If $w$ is a nontrivial solution of (3.14) with $w(a)=0$, then

$$
R\left\{\bar{w}(b) w^{\prime}(b)\right\}=e^{-b^{2}} \int_{a}^{b} e^{x^{2}}\left|w^{\prime}\right|^{2} d x>0,
$$

where $R(z)$ denotes the real part of the complex number $z$. We conclude that $w$ has $a$ as its only real root and that $w^{\prime}$ has no real roots at all.

Now suppose that (3.12) does not hold for some $a \neq b$. Then we can find a nontrivial pair of constants $A$ and $B$ such that

$$
w(x)=A \theta_{1}(x)+B \theta_{2}(x)
$$

has real roots at $a$ and $b$. By differentiating under the integral sign it is easy to verify that $w$, as defined in (3.15), is a solution of (3.14). Hence, by the previous argument, $w \equiv 0$. But this is clearly impossible since, for $x=0$, the Wronskian of $\theta_{1}$ and $\theta_{2}$ is simply

$$
-2 \theta_{1}(0) \theta_{1}^{\prime}(0)=-\Gamma\left(\frac{1+i \mu}{2}\right) \Gamma\left(1+\frac{i \mu}{2}\right) \neq 0 .
$$

Similarly if (3.13) does not hold we can find a nontrivial pair of constants $A$ and $B$ such that $w$, as defined in (3.15), has a root at $a$ and such that $w^{\prime}$ has a root at $b$. Again this implies that $w \equiv 0$ and we obtain a contradiction as before.

Finally we require the following partial converse for Corollary 1.

Lemma 5. Suppose that $u \in H^{+}$in $0<t<c$. If, for some path $\gamma \subset P\left(a, x_{0}\right)$,

$$
u(x, t)=O(1)
$$

as $(x, t) \rightarrow\left(x_{0}, 0\right),(x, t) \in \gamma$, then 


$$
\frac{\alpha\left(x_{0}+x\right)-\alpha\left(x_{0}\right)}{x}=O(1)
$$

as $x \rightarrow 0$. Suppose that $u \in H^{+}$in $0<t<\infty$. If, for some path $\gamma \subset P\left(a, x_{0}\right),(3.16)$ holds as $t \rightarrow \infty,(x, t) \in \gamma$, then (3.17) holds as $|x| \rightarrow \infty$.

Proof. Assume $x_{0}=0$. Then $(x, t) \in P(a, 0)$ and $|y| \leqq t^{1 / 2}$ imply that

$$
k(x-y, t) \geqq \frac{1}{(4 \pi t)^{1 / 2}} e^{-(1+a)^{2} / 4}=\frac{C_{4}}{2 t^{1 / 2}}
$$

where $C_{4}=C_{4}(a)>0$. Since $u \in H^{+}, \alpha$ is nondecreasing and we obtain

$$
0 \leqq \frac{\alpha\left(t^{1 / 2}\right)-\alpha\left(-t^{1 / 2}\right)}{2 t^{1 / 2}} \leqq \frac{1}{C_{4}} \int_{-t^{1 / 2}}^{t^{1 / 2}} k(x-y, t) d \alpha(y) \leqq \frac{u(x, t)}{C_{4}}
$$

for $(x, t) \in \gamma \subset P(a, 0)$. Both parts of the lemma follow from (3.16) and (3.18).

We consider now the proofs for Theorem 5 and Theorem 6.

Proof of Theorem 5. We begin with the first part of Theorem 5. Let $\alpha\left(x_{0}\right)=0, x_{0}=0$, and, for convenience, replace $a$ by $2 a$ and $b$ by $2 b$ in (3.7). Then we can write

$$
\begin{aligned}
u\left(2 a t^{1 / 2}, t\right) & =\frac{1}{(4 \pi t)^{1 / 2}} \int_{-\infty}^{\infty} e^{-\left[(y / 2 t]^{2}\right)-a^{1 / 2}} d \alpha(y)=\frac{1}{s} \frac{1}{\pi^{1 / 2}} \int_{-\infty}^{\infty} e^{-[(y / s)-a]^{2}} d \alpha(y) \\
& =\frac{1}{s} \int_{0}^{\infty} f_{1}(y / s) d \alpha(y)+\frac{1}{s} \int_{0}^{\infty} f_{2}(y / s) d \beta(y),
\end{aligned}
$$

where $s=2 t^{1 / 2}, \beta(x)=-\alpha(-x)$, and

$$
f_{1}(x)=\frac{1}{\pi^{1 / 2}} e^{-(x-a)^{2}}, \quad f_{2}(x)=\frac{1}{\pi^{1 / 2}} e^{-(x+a)^{2}} .
$$

Similarly, we can write

$$
u\left(2 b t^{1 / 2}, t\right)=\frac{1}{s} \int_{0}^{\infty} g_{1}(y / s) d \alpha(y)+\frac{1}{s} \int_{0}^{\infty} g_{2}(y / s) d \beta(y),
$$

where $s$ and $\beta$ are as above and where

$$
g_{1}(x)=\frac{1}{\pi^{1 / 2}} e^{-(x-b)^{2}}, \quad g_{2}(x)=\frac{1}{\pi^{1 / 2}} e^{-(x+b)^{2}} .
$$

The hypotheses (3.7) now imply that

$$
\begin{aligned}
& \frac{1}{s} \int_{0}^{\infty} f_{1}(y / s) d \alpha(y)+\frac{1}{s} \int_{0}^{\infty} f_{2}(y / s) d \beta(y) \rightarrow A, \\
& \frac{1}{s} \int_{0}^{\infty} g_{1}(y / s) d \alpha(y)+\frac{1}{s} \int_{0}^{\infty} g_{2}(y / s) d \beta(y) \rightarrow A
\end{aligned}
$$


as $s \rightarrow 0+$. To complete the proof we will show that Lemma 3 can be applied to $(3.19)$.

Since $f_{1}, f_{2}, g_{1}, g_{2}$ are continuous in $0 \leqq x<\infty$ and are $O\left(1 / x^{2}\right)$ for large $x$, it follows that all of these functions are in $M$ [8, p. 299]. Obviously $\alpha$ and $\beta$ are monotone and $\alpha(0)=\beta(0)=0$. From (3.7) and Lemma 5 we obtain

$$
\begin{aligned}
& \int_{x}^{2 x} \frac{|d \alpha(y)|}{y} \leqq \frac{\alpha(2 x)-\alpha(x)}{x}=O(1), \\
& \int_{x}^{2 x} \frac{|d \beta(y)|}{y} \leqq \frac{\alpha(-x)-\alpha(-2 x)}{x}=O(1)
\end{aligned}
$$

for $0<x \leqq d$, and, appealing to (2.2), we see we can assume that (3.20) holds for $0<x<\infty$. Hence $\alpha, \beta \in V$. Using (3.10),

$$
\begin{array}{ll}
F_{1}(\mu)=\frac{1}{\pi^{1 / 2}} \theta_{1}(a), & F_{2}(\mu)=\frac{1}{\pi^{1 / 2}} \theta_{2}(a), \\
G_{1}(\mu)=\frac{1}{\pi^{1 / 2}} \theta_{1}(b), & G_{2}(\mu)=\frac{1}{\pi^{1 / 2}} \theta_{2}(b),
\end{array}
$$

for each real $\mu$, where $\theta_{1}$ and $\theta_{2}$ are as defined in Lemma 4 , and hence

$$
F_{1}(\mu) G_{2}(\mu)-F_{2}(\mu) G_{1}(\mu) \neq 0
$$

by (3.12). Finally since

$$
F_{1} G_{2}-F_{2} G_{1}=F_{1}-G_{1}=G_{2}-F_{2},
$$

we can apply Lemma 3 to (3.19) to conclude that

$$
\frac{\alpha(x)}{x} \rightarrow A, \quad \frac{-\alpha(-x)}{x}=\frac{\beta(x)}{x} \rightarrow A
$$

as $x \rightarrow 0+$, and hence that $\alpha^{\prime}(0)=A$.

For the second part of Theorem 5 proceed as before. The hypothesis (3.7) now implies that (3.19) holds as $s \rightarrow \infty$, and, with Lemma 5, we obtain (3.20) for $d \leqq x<\infty$. Then (2.3) allows us to assume that (3.20) is valid for $0<x<\infty$ and again $\alpha, \beta \in V$. The proof is then completed by appealing to the $s, x \rightarrow \infty$ part of Lemma 3.

Proof for Theorem 6. Let $\alpha\left(x_{0}\right)=0, x_{0}=0$, and replace $a$ by $2 a$ and $b$ by $2 b$ in (3.9). Then arguing as before and differentiating under the integral sign in (1.1) we obtain

$$
\begin{aligned}
u\left(2 a t^{1 / 2}, t\right) & =\frac{1}{s} \int_{0}^{\infty} f_{1}(y / s) d \alpha(y)+\frac{1}{s} \int_{0}^{\infty} f_{2}(y / s) d \beta(y), \\
t^{1 / 2} u_{x}\left(2 b t^{1 / 2}, t\right) & =\frac{1}{s} \int_{0}^{\infty} g_{1}(y / s) d \alpha(y)+\frac{1}{s} \int_{0}^{\infty} g_{2}(y / s) d \beta(y),
\end{aligned}
$$


where $s=2 t^{1 / 2}, \beta(x)=-\alpha(x)$, and where

$$
\begin{array}{ll}
f_{1}(x)=\frac{1}{\pi^{1 / 2}} e^{-(x-a)^{2}}, & f_{2}(x)=\frac{1}{\pi^{1 / 2}} e^{-(x+a)^{2}}, \\
g_{1}(x)=\frac{x-b}{\pi^{1 / 2}} e^{-(x-b)^{2}}, & g_{2}(x)=-\frac{x+b}{\pi^{1 / 2}} e^{-(x+b)^{2}} .
\end{array}
$$

The hypotheses for the first part of Theorem 6 then imply that

$$
\begin{aligned}
& \frac{1}{s} \int_{0}^{\infty} f_{1}(y / s) d \alpha(y)+\frac{1}{s} \int_{0}^{\infty} f_{2}(y / s) d \beta(y) \rightarrow A, \\
& \frac{1}{s} \int_{0}^{\infty} g_{1}(y / s) d \alpha(y)+\frac{1}{s} \int_{0}^{\infty} g_{2}(y / s) d \beta(y) \rightarrow 0
\end{aligned}
$$

as $s \rightarrow 0+$. Now $f_{1}, f_{2}, g_{1}, g_{2}$ are in $M, \alpha$ and $\beta$ are monotone with $\alpha(0)=\beta(0)=0$, and the argument of Theorem 5 shows we can assume that $\alpha$ and $\beta$ are in $V$. Since

$$
\begin{array}{ll}
F_{1}(\mu)=\frac{1}{\pi^{1 / 2}} \theta_{1}(a), & F_{2}(\mu)=\frac{1}{\pi^{1 / 2}} \theta_{2}(a), \\
G_{1}(\mu)=\frac{1}{2 \pi^{1 / 2}} \theta_{1}^{\prime}(b), & G_{2}(\mu)=\frac{1}{2 \pi^{1 / 2}} \theta_{2}^{\prime}(b),
\end{array}
$$

we see from (3.13) that

$$
F_{1}(\mu) G_{2}(\mu)-F_{2}(\mu) G_{1}(\mu) \neq 0
$$

for all real $\mu$. Hence we can apply Lemma 3 to (3.22) and, since

$$
F_{1} G_{2}-F_{2} G_{1}=G_{2}=-G_{1}
$$

we conclude that (3.21) holds as $x \rightarrow 0+$. This completes the proof for the first part of Theorem 6. The proof for the second part follows similarly.

4. Additional remarks. In the first part of Theorem 4 we ask that $u \rightarrow A$ as $(x, t) \rightarrow\left(x_{0}, 0\right)$ along one ray. In the first part of Theorem 5 we ask that $u \rightarrow A$ as $(x, t) \rightarrow\left(x_{0}, 0\right)$ along two different parabolic arcs, i.e. the curves

$$
x=x_{0}+a t^{1 / 2}, \quad x=x_{0}+b t^{1 / 2}, \quad a \neq b .
$$

The following example shows that two arcs are required in the hypotheses of Theorem 5 .

Theorem 7. Given any fixed number $a$, there exists a bounded $u \in H^{+}$in $0<t<\infty$ such that

$$
u\left(a t^{1 / 2}, t\right)=1
$$

for $0<t<\infty$ and such that, for each $b \neq a$, 
$\lim \inf u\left(b t^{1 / 2}, t\right)<\lim \sup u\left(b t^{1 / 2}, t\right)$

as $t \rightarrow 0+$ and as $t \rightarrow \infty$.

Proof. For convenience replace $a$ by $2 a$ and $b$ by $2 b$. Then let $f_{1}, f_{2}, g_{1}, g_{2}$ be as defined in the proof for Theorem 5 and, using (3.10), let

$$
\begin{array}{r}
\alpha(x)=x+R\left\{F_{2}(\mu) \frac{x^{i \mu+1}}{i \mu+1}\right\}, \\
-\alpha(-x)=x-R\left\{F_{1}(\mu) \frac{x^{i \mu+1}}{i \mu+1}\right\}
\end{array}
$$

for $0 \leqq x<\infty$, where $\mu \neq 0$ and where $R(z)$ again denotes the real part of the complex number $z$. Since

$$
\begin{aligned}
& \left|F_{1}(\mu)\right| \leqq \frac{1}{\pi^{1 / 2}} \int_{0}^{\infty} e^{-(x-a)^{2}} d x<1, \\
& \left|F_{2}(\mu)\right| \leqq \frac{1}{\pi^{1 / 2}} \int_{0}^{\infty} e^{-(x+a)^{2}} d x<1,
\end{aligned}
$$

we see that $0<\alpha^{\prime}(x)<2$ for $x \neq 0$, and hence that

$$
u(x, t)=\int_{-\infty}^{\infty} k(x-y, t) d \alpha(y)=\int_{-\infty}^{\infty} k(x-y, t) \alpha^{\prime}(y) d y
$$

is bounded and in $H^{+}$over $0<t<\infty$. Following the proof for Theorem 5 we have

$$
\begin{aligned}
u\left(2 a t^{1 / 2}, t\right) & =\frac{1}{s} \int_{0}^{\infty} f_{1}(y / s) d \alpha(y)-\frac{1}{s} \int_{0}^{\infty} f_{2}(y / s) d \alpha(-y) \\
& =1+R\left\{\frac{F_{2}(\mu)}{s} \int_{0}^{\infty} f_{1}(y / s) y^{i \mu} d y-\frac{F_{1}(\mu)}{s} \int_{0}^{\infty} f_{2}(y / s) y^{i \mu} d y\right\} \\
& =1+R\left\{s^{i \mu}\left[F_{2}(\mu) F_{1}(\mu)-F_{1}(\mu) F_{2}(\mu)\right]\right\}=1
\end{aligned}
$$

for $0<t<\infty, s=2 t^{1 / 2}$. Similarly

$$
\begin{aligned}
u\left(2 b t^{1 / 2}, t\right) & =\frac{1}{s} \int_{0}^{\infty} g_{1}(y / s) d \alpha(y)-\frac{1}{s} \int_{0}^{\infty} g_{2}(y / s) d \alpha(-y) \\
& =1+R\left\{s^{i \mu}\left[F_{2}(\mu) G_{1}(\mu)-F_{1}(\mu) G_{2}(\mu)\right]\right\}
\end{aligned}
$$

for $0<t<\infty$. Since $b \neq a,(3.12)$ yields

$$
H(\mu)=F_{2}(\mu) G_{1}(\mu)-F_{1}(\mu) G_{2}(\mu) \neq 0,
$$

and we conclude that 


$$
\lim \sup u\left(2 b t^{1 / 2}, t\right)-\lim \inf u\left(2 b t^{1 / 2}, t\right)=2|H(\mu)|>0
$$

as $t \rightarrow 0+$ and as $t \rightarrow \infty$. This completes the proof for Theorem 7 .

It is clear, in the above example, that $\alpha^{\prime}(0)$ does not exist and that $\alpha(x) / x$ does not have a limit as $|x| \rightarrow \infty$. Moreover, if we fix $a \neq 0$, it is easy to see that $D \alpha(0)$ does not exist and that

$$
\frac{\alpha(x)-\alpha(-x)}{2 x}
$$

does not have a limit as $x \rightarrow \infty$. Hence we conclude that, when $a \neq 0$, the hypothesis

$$
u\left(x_{0}+a t, t\right) \rightarrow A \text { as } t \rightarrow 0+,
$$

in the first part of Theorem 4, cannot be replaced by the hypothesis

$$
u\left(x_{0}+a t^{1 / 2}, t\right) \rightarrow A \text { as } t \rightarrow 0+.
$$

When $E$ is any set in the $x t$-plane, we let $E_{c}$ denote the part of $E$ which is contained in the half plane $t \leqq c$. If we make use of the following maximum principle (see [7] or [17]) for functions in $H$, we can replace the two parabolic arcs in Theorem 5 by more general curves.

Lemma 6. Suppose that $u \in H$ in a bounded domain $\delta$ with boundary $\gamma$. Given any $c$, if

$$
\limsup _{(x, t) \rightarrow\left(x_{1}, t_{1}\right)} u(x, t) \leqq A \quad(x, t) \in \delta_{c},
$$

for all $\left(x_{1}, t_{1}\right) \in \gamma_{c}$, then $u \leqq A$ in $\delta_{c}$.

Here, and in what follows, we let $D=D\left(x_{0}\right)$ denote an infinite domain bounded by two disjoint arcs in $0<t<\infty$ which terminate at the point $\left(x_{0}, 0\right)$ and which cross the line $t=c$ for each $0<c<\infty$. We assume further that $D_{c}$ is bounded for each such $c$ and we let $\Gamma=\Gamma\left(x_{0}\right)$ denote the boundary for $D$.

Theorem 8. Suppose that $u \in H$ in $0<t<c$ and that, for some $0<b<c, u$ is bounded in $D_{b}$. If

$$
u(x, t) \rightarrow A
$$

as $(x, t) \rightarrow\left(x_{0}, 0\right),(x, t) \in \Gamma$, then (4.1) holds as $(x, t) \rightarrow\left(x_{0}, 0\right),(x, t) \in D$. Suppose that $u \in H$ in $0<t<\infty$ and that, for some $b>0, u$ is bounded in $D-D_{b}$. If (4.1) holds as $t \rightarrow \infty,(x, t) \in \Gamma$, then (4.1) holds as $t \rightarrow \infty,(x, t) \in D$.

Proof. Consider the first part. For each $\epsilon>0$ we can find $a>0$ such that $|u-A|<M$ in $D_{a}$ and such that

$$
|u(x, t)-A| \leqq \epsilon
$$

for $(x, t) \in \Gamma_{a},(x, t) \neq\left(x_{0}, 0\right)$. Next for each $d>0$ let 


$$
v(x, t)=u(x, t)-A-M \int_{\left|y-x_{0}\right| \leqq d} k(x-y, t) d y .
$$

Clearly $v \in H$ in $0<t<c$. From (2.2) of Lemma 1 we see that

$$
\int_{\left|y-x_{0}\right| \leq d} k(x-y, t) d y \rightarrow 1
$$

as $(x, t) \rightarrow\left(x_{0}, 0\right), t>0$, and, with (4.2), we obtain

$$
\lim _{(x, t) \rightarrow\left(x_{1}, t_{1}\right)} v(x, t) \leqq \epsilon \quad(x, t) \in D_{a},
$$

for all $\left(x_{1}, t_{1}\right) \in \Gamma_{a}$. Applying Lemma 6 to $v$ yields

$$
u(x, t) \leqq A+\epsilon+M \int_{\left|y-x_{0}\right| \leqq d} k(x-y, t) d y
$$

for $(x, t) \in D_{a}$, and letting $d \rightarrow 0+$ we obtain $u \leqq A+\epsilon$ in $D_{a}$. A similar argument gives $u \geqq A-\epsilon$ in $D_{a}$ thus completing the proof for the first part of Theorem 8 .

For the second part pick $a>0$ so that $|u-A|<M$ in $D-D_{a}$ and so that (4.2) holds for all $(x, t) \in \Gamma-\Gamma_{a}$. Let $\gamma$ denote the boundary for $D-D_{a}$. Since $D_{a}$ is bounded we can find a $d>0$ such that $\gamma_{a}$, the part of $\gamma$ contained in the line $t=a$, lies between the lines $x= \pm d$. Now set

$$
v(x, t)=u(x, t)-A-M \int_{|y| \leqq d} k(x-y, t-a) d y .
$$

Then $v \in H$ in $a<t<\infty$ and, again with (2.2), we see that for each $\left|x_{1}\right|<d$,

$$
\int_{|y| \leqq d} k(x-y, t-a) d y \rightarrow 1
$$

as $(x, t) \rightarrow\left(x_{1}, a\right), t>a$. Since $\gamma=\gamma_{a} \cup\left(\Gamma-\Gamma_{a}\right)$ we conclude that

$$
\limsup _{(x, t) \rightarrow\left(x ., t_{1}\right)} v(x, t) \leqq \epsilon \quad(x, t) \in D-D_{a},
$$

for all $\left(x_{1}, t_{1}\right) \in \gamma$. If we apply Lemma 6 to $v$ over $D_{c}-D_{a}, a<c<\infty$, and then let $c \rightarrow \infty$, we obtain

$$
u(x, t) \leqq A+\epsilon+M \int_{|y| \leqq d} k(x-y, t-a) d y
$$

for $(x, t) \in D-D_{a}$. A similar argument yields

$$
u(x, t) \geqq A-\epsilon-M \int_{|y| \leqq d} k(x-y, t-a) d y
$$

for $(x, t) \in D-D_{a}$ and since, by $(2.4)$, 


$$
\left|\int_{|y| \leqq d} k(x-y, t-a) d y\right| \leqq \frac{d}{(\pi(t-a))^{1 / 2}} \rightarrow 0
$$

as $t \rightarrow \infty$, uniformly in $x,|u-A| \leqq 2 \epsilon$ in $D-D_{c}$ for some $c>a$. This completes the proof for the second part of Theorem 8 .

The Phragmén-Lindelöf type argument used in the proof of the first part of Theorem 8 can be combined with a familiar step-by-step argument to give the following variant of Lemma 6.

Corollary 3. Suppose that $u$ is bounded above and that $u \in H$ in a bounded domain $\delta$ with boundary $\gamma$. Given any $c$, if

$$
\limsup _{(x, t) \rightarrow\left(x_{1}, t_{1}\right)} u(x, t) \leqq A \quad(x, t) \in \delta_{c},
$$

for all but a finite set of $\left(x_{1}, t_{1}\right) \in \gamma_{c}$, then $u \leqq A$ in $\delta_{c}$.

We can now prove the following extension of Theorem 5 .

Theorem 9. Suppose that $D\left(x_{0}\right) \subset P\left(a, x_{0}\right)$, for some $a>0$, and that $D$ contains two parabolic arcs through $\left(x_{0}, 0\right)$. If $u \in H^{+}$in $0<t<c$ and if

$$
u(x, t) \rightarrow A
$$

as $(x, t) \rightarrow\left(x_{0}, 0\right),(x, t) \in \Gamma$, then $\alpha^{\prime}\left(x_{0}\right)=A$. If $u \in H^{+}$in $0<t<\infty$ and if (4.3) holds as $t \rightarrow \infty,(x, t) \in \Gamma$, then

$$
\frac{\alpha\left(x_{0}+x\right)-\alpha\left(x_{0}\right)}{x} \rightarrow A
$$

as $|x| \rightarrow \infty$.

Proof. The hypotheses for the first part of Theorem 9 imply that

$$
u(x, t)=O(1)
$$

as $(x, t) \rightarrow\left(x_{0}, 0\right)$ along some path in $P\left(a, x_{0}\right)$. From Lemma 5 and Corollary 1 we conclude that (4.4) holds as $(x, t) \rightarrow\left(x_{0}, 0\right),(x, t) \in P\left(a, x_{0}\right)$, and hence that $u$ is bounded in $D_{b}$ for some $0<b<c$. With Theorem 8 we see that (4.3) holds as $(x, t) \rightarrow\left(x_{0}, 0\right)$ along the two parabolic arcs in $D$ and the desired conclusion follows from Theorem 5 . The proof for the second part is very similar.

COROllary 4. If, in Theorem $9, u$ is bounded, we can drop the restriction that $D\left(x_{0}\right)$ lie in some $P\left(a, x_{0}\right)$.

This is an immediate consequence of the above argument since this restriction is used only in proving that $u$ is bounded in $D_{b}$ or in $D-D_{b}$. On the other hand, without boundedness it is clear that some such restriction is 
necessary. For if we let $D$ be the domain bounded by the curve $x^{4}=t$ and set $u(x, t)=k(x, t)$, then

$$
u(x, t) \rightarrow 0
$$

as $(x, t) \rightarrow(0,0),(x, t) \in \Gamma$. But the normalized $\alpha$ is constant except for a jump of 1 at $x=0$ and $\alpha^{\prime}(0)=\infty$.

We conclude this section with a pair of remarks on steady state temperatures which are trivial consequences of some of the preceding results.

Corollary 5. Suppose that $u \in H^{+}$in $0<t<\infty$. If, for some $x_{0}$,

$$
u\left(x_{0}, t\right) \rightarrow A
$$

as $t \rightarrow \infty$, then, for each $a>0$,

$$
u(x, t) \rightarrow A
$$

as $t \rightarrow \infty$, uniformly in $x$ in $\left|x-x_{0}\right|<a$.

Corollary 6. Suppose that $u \in H^{+}$in $0<t<\infty$ and that, for some $0<c<\infty$, $u(x, c)$ is monotone in $x$. If, for some $x_{0}$,

$$
u\left(x_{0}, t\right)=O(1)
$$

as $t \rightarrow \infty$, then there exists a constant $A$ such that, for each $a>0,(4.6)$ holds as $t \rightarrow \infty$, uniformly in $x$ in $\left|x-x_{0}\right|<a$.

Proof for Corollary 5. From (4.5), Lemma 5, and Corollary 1 we see that, for each $a>0$,

$$
u_{x}(x, t)=O\left(\frac{1}{t^{1 / 2}}\right)
$$

as $t \rightarrow \infty,(x, t) \in P\left(a, x_{0}\right)$. Thus when $\left|x-x_{0}\right|<a, t>1$, we have

$$
\left|u(x, t)-u\left(x_{0}, t\right)\right| \leqq \frac{C_{5}}{t^{1 / 2}},
$$

where $C_{5}=C_{5}(a, u)$, and the desired conclusion follows from (4.5).

Proof for Corollary 6. Let $x_{0}=0$ and set $v(x, t)=u(x, t+c)$. Then $v \in H^{+}$ in $0<t<\infty$ and, with (1.3),

$$
v(x, t)=\int_{-\infty}^{\infty} k(x-y, t) d \beta(y), \quad \beta(x)=\int_{0}^{x} u(y, c) d y .
$$

If $u(x, c)$ is nondecreasing or nonincreasing in $x$, it follows that $\beta(x) / x$ is nondecreasing or nonincreasing, respectively, in $x, x \neq 0$. Next (4.7) and Lemma 5 imply that $\beta(x) / x$ is bounded as $|x| \rightarrow \infty$. We conclude that

$$
\frac{\beta(x)-\beta(-x)}{2 x}
$$


has a finite limit $A$ as $x \rightarrow \infty$ and hence, with Theorem 1, that

$$
u(0, t) \rightarrow A
$$

as $t \rightarrow \infty$. The rest follows from Corollary 5 .

In Corollary 6 we replace (4.5) by the weaker (4.7) and ask that the temperature be monotone at some time $t=c$. Some such additional condition is necessary. For let $u$ be the bounded function of Theorem 7 with $a \neq 0$ and $b=0$. Then (4.7) is satisfied,

$$
\liminf _{t \rightarrow \infty} u(0, t)<\limsup _{t \rightarrow \infty} u(0, t),
$$

and, with Corollary 5 , we see that

$$
\liminf _{t \rightarrow \infty} u\left(x_{0}, t\right)<\limsup _{t \rightarrow \infty} u\left(x_{0}, t\right)
$$

for all $x_{0}$.

5. Uniqueness theorems. We consider here some one-sided uniqueness theorems for functions in $H$ over $0<t<c$. We begin with the following preliminary result.

Lemma 7. Suppose that $\alpha$ has bounded variation over each finite interval, that

$$
\alpha(x-0) \geqq \alpha(x) \geqq \alpha(x+0)
$$

for all $x$, and that $\alpha^{\prime}(x)<\infty$ for all $x$, except an enumerable set, at which the derivative exists. Then

$$
\alpha\left(x_{2}\right)-\alpha\left(x_{1}\right) \leqq \int_{x_{1}}^{x_{2}} \alpha^{\prime}(x) d x
$$

for all $x_{1}, x_{2}, x_{1}<x_{2}$.

Proof. This is an immediate consequence of the de la Vallee Poussin decomposition theorem [16, p. 127]. For let $I$ denote the open interval $x_{1}<x<x_{2}$, let $X$ denote the points in $I$ where $\alpha$ is continuous, and let

$$
E_{\infty} \equiv\left\{x: \alpha^{\prime}(x)=\infty, x \in X\right\}, \quad E_{-\infty} \equiv\left\{x: \alpha^{\prime}(x)=-\infty, x \in X\right\} .
$$

If $\mu=\mu(E)$ denotes the signed measure corresponding to $\alpha$, then the de la Vallée Poussin theorem yields

$$
\mu(X)=\mu\left(E_{\infty}\right)+\mu\left(E_{-\infty}\right)+\int_{X} \alpha^{\prime}(x) d x .
$$

By hypothesis $E_{\infty}$ is at most enumerable and hence $\mu\left(E_{\infty}\right)=0$. Clearly $\mu\left(E_{-\infty}\right)$ $\leqq 0$ and, from (5.1), we see that

$$
\mu(I)=\mu(I-X)+\mu(X) \leqq \mu(X) .
$$

Since $I-X$ is enumerable we can combine (5.1), (5.2), and (5.3) to obtain 


$$
\alpha\left(x_{2}\right)-\alpha\left(x_{1}\right) \leqq \mu(I) \leqq \int_{x_{1}}^{x_{2}} \alpha^{\prime}(x) d x
$$

as desired.

The following theorem extends results of Rosenbloom [15] and Widder [19] and is a one-sided analogue of a theorem on harmonic functions due to Lohwater [11].

Theorem 10. Suppose that $u \in H^{\Delta}$ in $0<t<c$. If

$$
\lim _{t \rightarrow 0+} u(x, t)<\infty
$$

for all $x$ at which this limit exists and if $\left(^{3}\right)$

$$
\lim _{t \rightarrow 0+} u(x, t) \leqq A
$$

for almost all $x$, then $u \leqq A$ in $0<t<c$.

Proof. If $\alpha$ has a jump of $\lambda$ at $x=x_{0}$, then, by Lemma 2,

$$
u\left(x_{0}, t\right) \sim \frac{\lambda}{(4 \pi t)^{1 / 2}}
$$

as $t \rightarrow 0+$, and, with (5.4), it follows that $\lambda<0$. Hence, assuming that $\alpha$ is normalized, (5.1) holds for all $x$. Next suppose that $\alpha$ has a derivative, finite or infinite, at $x_{0}$. Then, with Theorem 2 and Corollary 2, we see that

$$
\lim _{t \rightarrow 0+} u\left(x_{0}, t\right)=\alpha^{\prime}\left(x_{0}\right),
$$

and we conclude from (5.4) that $\alpha^{\prime}(x)<\infty$ for all $x$ at which this derivative exists. From (5.5) we obtain $\alpha^{\prime}(x) \leqq A$ for almost all $x$. Lemma 7 yields

$$
\alpha\left(x_{2}\right)-\alpha\left(x_{1}\right) \leqq \int_{x_{1}}^{x_{2}} \alpha^{\prime}(x) d x \leqq A\left(x_{2}-x_{1}\right)
$$

for all $x_{1}, x_{2}, x_{1}<x_{2}$, and we conclude that

$$
u(x, t)=\int_{-\infty}^{\infty} k(x-y, t) d \alpha(y) \leqq A \int_{-\infty}^{\infty} k(x-y, t) d y=A
$$

in $0<t<c$.

The following is an immediate consequence of Theorem 10 .

Corollary 7. Suppose that $u \in H^{\Delta}$ in $0<t<c$. If

$$
\lim _{t \rightarrow 0+} u(x, t)=A
$$

( ${ }^{3}$ Since $\alpha$ has a finite derivative almost everywhere, the limit in (5.5) exists for almost all $x$ by virtue of Theorem 2 . 
for all $x$, then $u \equiv A$ in $0<t<c$.

Theorem 10 can also be used to obtain the following one-sided version of a well known theorem due to Tychonoff [18].

TheOREM 11. Suppose that $u \in H$ in $0<t<c$. If

$$
u(x, t) \leqq M e^{a x^{2}},
$$$$
M>0, a>0,
$$

for all $x, 0<t<c$, and if

$$
\liminf _{t \rightarrow 0+} u(x, t) \leqq A
$$

for almost all $x$, then $u \leqq A$ in $0<t<c$.

Proof. Let $b=\operatorname{Min}(1 / 4 a, c)$ and let

$$
v(x, t)=\frac{M}{(1-4 a t)^{1 / 2}} e^{a x^{2} /(1-4 a t)} .
$$

Then $v, v-u \in H^{+}$in $0<t<b$. Hence $u \in H^{\Delta}$ in this strip and, with (5.7),

$$
\lim _{t \rightarrow 0+} u(x, t)=\liminf _{t \rightarrow 0+} u(x, t) \leqq A
$$

for almost all $x$. From (5.6) we see that

$$
\lim _{t \rightarrow 0+} u(x, t) \leqq M e^{a x^{2}}<\infty
$$

for all $x$ at which this limit exists and, appealing to Theorem 10, we conclude that $u \leqq A$ in $0<t<b$. If $b<c$, the proof is completed by a familiar step-bystep argument [18].

Corollary 8. Suppose that $u \in H$ in $0<t<c$. If (5.6) holds for all $x$, $0<t<c$, and if

$$
\lim _{t \rightarrow 0+} u(x, t)=A
$$

for all $x$, then $u \equiv A$ in $0<t<c$.

Proof. With Theorem 11 we see that $u \leqq A$ in $0<t<c$. Hence $A-u \in H^{+}$ and $u \in H^{\Delta}$ in this strip, and Corollary 7 yields $u \equiv A$.

We have next the following one-sided version of a recent theorem due to Birkhoff and Kotik [1].

Theorem 12. Suppose that $u \in H$ in $0<t<c$. If

$$
\int_{x_{1}}^{x_{2}} u(y, t) d y \leqq M e^{a\left(x_{1}^{2}+x_{2}^{2}\right)}, \quad M>0, a>0,
$$

for all $x_{1}, x_{2}, x_{1}<x_{2}, 0<t<c$, and if 


$$
\liminf _{t \rightarrow 0+} \int_{x_{1}}^{x_{2}} u(y, t) d y \leqq A\left(x_{2}-x_{1}\right)
$$

for almost all $x_{1}, x_{2}, \quad x_{1}<x_{2}$, then $u \leqq A$ in $0<t<c$.

Proof. By the last hypothesis we mean that (5.10) holds for all $x_{1}, x_{2} \notin E$, $x_{1}<x_{2}$, where $E$ is a set of measure zero in the $x$-axis. For each $h>0$ let

$$
v_{h}(x, t)=\frac{1}{2 h} \int_{-h}^{h} u(x+y, t) d y .
$$

Then $v_{h} \in H$ in $0<t<c$ and, from (5.9), we see that

$$
v_{h}(x, t) \leqq N e^{b x^{2}}
$$

for all $x, 0<t<c$, where $N=(1 / 2 h) M e^{2 a h^{2}}, b=2 a$. From (5.10) it follows that

$$
\liminf _{t \rightarrow 0+} v_{h}(x, t) \leqq A
$$

for almost all $x$ and, appealing to Theorem 11 , we conclude that $v_{h} \leqq A$ in $0<t<c$. Finally

$$
u(x, t)=\lim _{h \rightarrow 0+} v_{h}(x, t) \leqq A
$$

in $0<t<c$ as desired.

With the aid of Theorem 12 we obtain the following extension of the above mentioned theorem of Birkhoff and Kotik.

Theorem 13. Suppose that $u \in H$ in $0<t<c$. If (5.9) holds for all $x_{1}, x_{2}$, $x_{1}<x_{2}, 0<t<c$, and if

$$
\lim _{t \rightarrow 0+} \int_{x_{1}}^{x_{2}} u(y, t) d y=A\left(x_{2}-x_{1}\right)
$$

for almost all $x_{1}, x_{2}$, then $u \equiv A$ in $0<t<c$.

In the Birkhoff-Kotik theorem, the one-sided restriction (5.9) is replaced by a two-sided condition equivalent to the following:

$$
\left|\int_{x_{1}}^{x_{2}} u(y, t) d y\right| \leqq M e^{a\left(x_{1}^{2}+x_{2}^{2}\right)}, \quad M>0, a>0,
$$

for all $x_{1}, x_{2}, 0<t<c$.

Proof. By Theorem 12 we see that $u \leqq A$ in $0<t<c$ and hence that $u \in H^{\Delta}$ in this strip. Next if we assume, as we may, that $\alpha$ is normalized, then (1.3) and (5.11) yield

$$
\alpha\left(x_{2}\right)-\alpha\left(x_{1}\right)=\lim _{t \rightarrow 0+} \int_{x_{1}}^{x_{2}} u(y, t) d y=A\left(x_{2}-x_{1}\right)
$$


for almost all $x_{1}, x_{2}$. Since (5.12) holds for all $x_{1}, x_{2} \in E$, where $E$ is dense in the $x$-axis, we conclude from a limiting argument that

$$
\alpha\left(x_{2}\right)-\alpha\left(x_{1}\right)=A\left(x_{2}-x_{1}\right)
$$

for all $x_{1}, x_{2}$, and hence that $u \equiv A$ in $0<t<c$.

The following is an immediate consequence of the above argument.

Corollary 9. Suppose that $u \in H^{\Delta}$ in $0<t<c$ and that $E$ is a set dense in the $x$-axis. If

$$
\lim _{t \rightarrow 0+} \int_{x_{1}}^{x_{2}} u(y, t) d y \leqq A\left(x_{2}-x_{1}\right)
$$

for all $x_{1}, x_{2} \in E, x_{1}<x_{2}$, then $u \leqq A$ in $0<t<c$.

We require next the following variant of the parabolic maximum principle, Lemma 6.

Lemma 8. Suppose that $u \in H$ in $0<t<c$ and that $\delta$ is the rectangle bounded by the lines $x=x_{1}, x=x_{2}, t=0, t=c$, where $x_{1}<x_{2}$. If

$$
u\left(x_{1}, t\right) \leqq A, \quad u\left(x_{2}, t\right) \leqq A
$$

for $0<t<c$, and if, for each non-negative continuous function $f(x)$,

$$
\liminf _{t \rightarrow 0+} \int_{x_{1}}^{x_{2}} f(y) u(y, t) d y \leqq A \int_{x_{1}}^{x_{2}} f(y) d y,
$$

then $u \leqq A$ in $\delta$.

Proof. We can obviously assume that $A=0$. Next fix $(x, t) \in \delta$ and pick $\epsilon>0$ so that $0<t+\epsilon<c$. Then we can write

$$
\begin{aligned}
u(x, t+\epsilon)=\int_{x_{1}}^{x_{2}} G(x, t ; y, 0) u(y, \epsilon) d y & +\int_{0}^{t} \frac{\partial G}{\partial y}\left(x, t ; x_{1}, s\right) u\left(x_{1}, s+\epsilon\right) d s \\
& -\int_{0}^{t} \frac{\partial G}{\partial y}\left(x, t ; x_{2}, s\right) u\left(x_{2}, s+\epsilon\right) d s
\end{aligned}
$$

where $G$ is the Green's function

$$
G(x, t ; y, s)=\frac{1}{2 l}\left\{\theta_{3}\left(\frac{x-y}{2 l}, \frac{t-s}{l^{2}}\right)-\theta_{3}\left(\frac{x+y-2 x_{1}}{2 l}, \frac{t-s}{l^{2}}\right)\right\},
$$

where $l=x_{2}-x_{1}$, and where $\theta_{3}$ is the Jacobi theta function

$$
\theta_{3}(x, t)=1+2 \sum_{n=1}^{\infty} e^{-n^{2} \pi^{2} t} \cos 2 n \pi x .
$$

$\left(\right.$ See $_{i}[5]$ or [9] and make a change of variables.) Since 


$$
\frac{\partial G}{\partial y}\left(x, t ; x_{1}, s\right) \geqq 0, \quad-\frac{\partial G}{\partial y}\left(x, t ; x_{2}, s\right) \geqq 0,
$$

we see from (5.13) that

$$
u(x, t+\epsilon) \leqq \int_{x_{1}}^{x_{2}} G(x, \imath ; y, 0) u(y, \epsilon) d y
$$

and, letting $\epsilon \rightarrow 0+$, we conclude from (5.14) that

$$
u(x, t) \leqq \liminf _{\epsilon \rightarrow 0+} \int_{x_{1}}^{x_{2}} G(x, t ; y, 0) u(y, \epsilon) d y \leqq 0
$$

as desired.

We consider next the following extension of a uniqueness theorem due to Cooper [2].

Theorem 14. Suppose that $u \in H$ in $0<t<c$ and that $\left\{x_{n}\right\}$ is a sequence, defined for $-\infty<n<\infty$, such that $x_{n} \rightarrow \infty(-\infty)$ as $n \rightarrow \infty(-\infty)$. If

$$
u\left(x_{n}, t\right) \leqq M e^{a x_{n}^{2}}, \quad M>0, a>0,
$$

for all $n, 0<t<c$, and if $u$ converges weakly to $A$, over each finite interval, as $t \rightarrow 0+$, then $u \equiv A$ in $0<t<c$.

In Cooper's theorem, the one-sided restriction (5.15) is replaced by a twosided condition, namely that

$$
\left|u\left(x_{n}, l\right)\right| \leqq M e^{a x_{n}^{2}}, \quad M>0, a>0,
$$

for all $n, 0<t<c$.

Proof. Again we can assume that $A=0$. Next let $b=\operatorname{Min}(1 / 4 a, c)$, define $v$ as in (5.8), and let $w=u-v$. If we pick $x_{m}<x_{n}$, then

$$
w\left(x_{m}, t\right) \leqq 0, \quad w\left(x_{n}, t\right) \leqq 0
$$

for $0<t<b$ and, by virtue of the weak convergence,

$$
\liminf _{t \rightarrow 0+} \int_{x_{m}}^{x_{n}} f(y) w(y, t) d y \leqq \lim _{t \rightarrow 0+} \int_{x_{m}}^{x_{n}} f(y) u(y, t) d y=0
$$

for all non-negative continuous functions $f(x)$. Lemma 8 yields $u \leqq v$ in the rectangle bounded by the lines $x=x_{m}, x=x_{n}, t=0, t=b$, and letting $x_{m} \rightarrow-\infty$, $x_{n} \rightarrow \infty$ we conclude that $u \leqq v$ in the strip $0<t<b$. Hence $u \in H^{\Delta}$ in this strip. The weak convergence also implies that

$$
\lim _{t \rightarrow 0+} \int_{y_{1}}^{y_{2}} u(y, t) d y=0
$$

for all $y_{1}, y_{2}$ and, with Corollary 9 , we obtain $u \equiv 0$ in $0<t<b$. If $b<c$, the proof is completed by a step-by-step argument. 
The preceding argument suggests still another one-sided variant of the Tychonoff theorem similar to Theorem 11.

Theorem 15. Suppose that $u \in H$ in $0<t<c$ and that $\left\{x_{n}\right\}$ is as defined in Theorem 14. If (5.15) holds for all $n, 0<t<c$, and if

$$
\limsup _{(y, t) \rightarrow(x, 0)} u(y, t) \leqq A
$$

for all $x$, then $u \leqq A$ in $0<t<c$.

Proof. Set $A=0$ and let $b, v$, and $w$ be as defined in the proof of Theorem 14. Next fix $x_{m}<x_{n}$; clearly (5.16) holds for $0<t<b$. Then (5.17) implies that

$$
\lim _{(y, t) \rightarrow(x, 0)} w(y, t) \leqq 0
$$

for all $x$ and, with Lemma 6 , we obtain $u \leqq v$ in the rectangle bounded by the lines $x=x_{m}, x=x_{n}, t=0, t=b$. Arguing as before we conclude that $u \in H^{\Delta}$ in $0<t<b$ and Theorem 10 yields $u \leqq A$ in this strip.

Corollary 10. Suppose that $u \in H$ in $0<t<c$ and that $\left\{x_{n}\right\}$ is as defined in Theorem 14. If (5.15) holds for all $n, 0<t<c$, and if

$$
\lim _{(y, t) \rightarrow(x, 0)} u(y, t)=A
$$

for all $x$, then $u \equiv A$ in $0<t<c$.

6. Fejer-Riesz inequality. We conclude this paper with a heat equation analogue of the following well known inequality [4] and [14].

Fejer-Riesz InEQUality. Suppose that $u=u(z)$ is harmonic in $|z|<1$. Then, for each $0<\rho<1$,

$$
\int_{-\rho}^{\rho}\left|u_{x}(x)\right| d x \leqq \frac{1}{2} \int_{-\pi}^{\pi}\left|u_{\theta}\left(\rho e^{i \theta}\right)\right| d \theta .
$$

This inequality has an important interpretation, namely that under any conformal mapping of the unit disk onto a Jordan domain, the length of the image of a diameter never exceeds one-half the length of the image of the unit circle.

The following result suggests a new interpretation for this inequality.

Theorem 16. Suppose that $u \in H^{\Delta}$ in $0<t<\infty$. Then, for each $0<c<\infty$,

$$
\begin{aligned}
& \int_{c}^{\infty}\left|u_{x}(0, t)\right| d t \leqq \frac{1}{2} \int_{0}^{\infty}|u(x, c)-u(-x, c)| d x \leqq \frac{1}{2} \int_{-\infty}^{\infty}|u(x, c)| d x, \\
& \int_{c}^{\infty}\left|u_{t}(0, t)\right| d t \leqq \frac{1}{2} \int_{0}^{\infty}\left|u_{x}(x, c)-u_{x}(-x, c)\right| d x \leqq \frac{1}{2} \int_{-\infty}^{\infty}\left|u_{x}(x, c)\right| d x .
\end{aligned}
$$


Proof. We begin by considering (6.1) in the special case where, for a fixed $y, u(x, t)=k(x-y, t)$. In particular we observe that, with $s=|y| / 2 t^{1 / 2}$,

$$
\int_{0}^{\infty}\left|k_{x}(y, t)\right| d t= \begin{cases}\frac{1}{\pi^{1 / 2}} \int_{0}^{\infty} e^{-s^{2}} d s=\frac{1}{2} & y \neq 0 \\ 0 & y=0 .\end{cases}
$$

For the general case fix $0<c<\infty$. Then, since $u \in H^{\Delta}$ in $0<t<\infty$, we can write

$$
u(x, t+c)=\int_{-\infty}^{\infty} k(x-y, t) u(y, c) d y,
$$

where the integral is absolutely convergent in $0<t<\infty$. Differentiating under the integral sign [19], we obtain

$$
u_{x}(x, t+c)=\int_{-\infty}^{\infty} k_{x}(x-y, t) u(y, c) d y,
$$

and, with (6.3) and the Fubini theorem, we conclude that

$$
\begin{aligned}
\int_{0}^{\infty}\left|u_{x}(0, t+c)\right| d t & =\int_{0}^{\infty}\left|\int_{-\infty}^{\infty} k_{x}(-y, t) u(y, c) d y\right| d t \\
& \leqq \int_{0}^{\infty}\left\{\int_{0}^{\infty}\left|k_{x}(y, t)\right| d t\right\}|u(y, c)-u(-y, c)| d y \\
& =\frac{1}{2} \int_{0}^{\infty}|u(y, c)-u(-y, c)| d y .
\end{aligned}
$$

Hence we obtain (6.1). For (6.2) we can differentiate (6.4) with respect to $x$ to obtain

$$
u_{t}(x, t+c)=\int_{-\infty}^{\infty} k_{x x}(x-y, t) u(y, c) d y .
$$

If $0<t_{1}, t_{2}<\infty$, we obtain

$$
k\left(x_{1}, t_{1}\right) k\left(x_{2}, t_{2}\right) \leqq\left(\frac{t_{1}+t_{2}}{4 \pi t_{1} t_{2}}\right)^{1 / 2} k\left(x_{1}+x_{2}, t_{1}+t_{2}\right)
$$

from [13] or direct calculation. Since

$$
|u(y, c)| \leqq \int_{-\infty}^{\infty} k(y-z, c)|d \alpha(z)|
$$

we conclude from (6.6), with $x_{1}=x-y, x_{2}=y-z, t_{1}=t, t_{2}=c$, that

$$
|u(y, c)| k(x-y, t) \leqq\left(\frac{c+t}{4 \pi c t}\right)^{1 / 2} \int_{-\infty}^{\infty} k(x-z, t+c)|d \alpha(z)|<\infty
$$


for each fixed $(x, t)$ in $0<t<\infty$. With (6.7) it is easy to show that

$$
u(y, c) k_{x}(x-y, t)=o(1)
$$

as $|y| \rightarrow \infty$, and integration by parts in (6.5) yields

$$
u_{t}(x, t+c)=\int_{-\infty}^{\infty} k_{x}(x-y, t) u_{y}(y, c) d y .
$$

The proof for (6.2) is then completed as before.

Both (6.1) and (6.2) have physical interpretations, if we think of $u$ as the temperature, on an absolute scale, of an infinite insulated rod with unit cross-section and unit thermal diffusivity. For (6.1), suppose that at time $t=c$ the total heat in the rod is finite and equal to $A$, i.e.

$$
\int_{-\infty}^{\infty} u(x, c) d x=A
$$

Then it follows that the amount of heat which crosses any fixed section of the rod, in the time interval $c \leqq t<\infty$, never exceeds $A / 2$. For (6.2), suppose that at time $t=c$ the temperature variation along the rod is bounded and equal to $A$. Then at each section of the rod the temperature variation in time, for $c \leqq t<\infty$, never exceeds $A / 2$.

\section{REFERENCES}

1. G. Birkhoff and J. Kotik, Note on the heat equation, Proc. Amer. Math. Soc. vol. 5 (1954) pp. 162-167.

2. J. L. B. Cooper, The uniqueness of the solution of the equation of heat conduction, J. London Math. Soc. vol. 25 (1950) pp. 173-180.

3. J. Czipszer, Sur la propagation de la chaleur dans une barre infinie, I, Magyar Tud. Akad. Alkalm. Mat. Int. Közl. vol. 3 (1954) pp. 395-408 (Hungarian, Russian and French summaries).

4. L. Fejêr and F. Riesz, Über einige funktionentheoretische Ungleichungen, Math. Z. vol. 11 (1921) pp. 305-314.

5. W. Fulks, On the unique determination of solutions of the heat equation, Pacific J. Math. vol. 3 (1953) pp. 387-391.

6. F. W. Gehring, The Fatou theorem and its converse, Trans. Amer. Math. Soc. vol. 85 (1957) pp. 106-121. Errata, ibid., vol. 86 (1957) p. 532.

7. - On solutions of the equation of heat conduction, Michigan Math. J. vol. 5 (1958) pp. 191-202.

8. G. H. Hardy, Divergent series, Oxford, 1949.

9. P. Hartman and A. Wintner, On solutions of the equation of heat conduction, Amer. J. Math. vol. 72 (1950) pp. 367-395.

10. E. L. Ince, Ordinary differential equations, New York, 1944.

11. A. J. Lohwater, $A$ uniqueness theorem for a class of harmonic functions, Proc. Amer. Math. Soc. vol. 3 (1952) pp. 278-279.

12. L. H. Loomis, The converse of the Fatou theorem for positive harmonic functions, Trans. Amer. Math. Soc. vol. 53 (1943) pp. 239-250.

13. D. Resch, Temperature bounds on the infinite rod, Proc. Amer. Math. Soc. vol. 3 (1952) pp. 632-634. 
14. F. Riesz, Eine Ungleichung für harmonische Funklionen, Monatshefte Math. u. Physik vol. 43 (1936) pp. 401-406.

15. P. C. Rosenbloom, Linear equations of parabolic type with constant coefficients, Annals of Mathematics Studies, no. 33, Princeton, 1954.

16. S. Saks, Theory of the integral, Warsaw, 1937.

17. S. Täcklind, Sur les classes quasianalytiques des solutions des équations aux dérivées partielles du type parabolique, Nova Acta Soc. Sci. Upsal. vol. 10 (1936) pp. 1-57.

18. A. Tychonoff, Théorèmes d'unicité pour l'équation de la chaleur, Rec. Math. (Mat. Sb.) vol. 42 (1935) pp. 199-216.

19. D. V. Widder, Positive temperatures on an infinite rod, Trans. Amer. Math. Soc. vol. 55 (1944) pp. 85-95.

20. — The Laplace transform, Princeton, 1946.

21. - Integral transforms related to heal conduction, Ann. Mat. Pura Appl. vol. 42 (1956) pp. 279-305.

UNIVERSITY OF MiChIGAN,

AnN Arbor, Michigan 